\title{
La equinoterapia como una alternativa terapéutica dirigida al tratamiento de la discapacidad infantil y su impacto en el proceso de rehabilitación.
}

The equine therapy like a therapeutic alternative directed to the treatment of the infantile disability and their impact in the process of Rehabilitation

José Díaz Valdés. ${ }^{1}$, Eugenio Rodolfo González Pérez. ${ }^{2}$, Luisa María Torres García. ${ }^{3}$ \& Luis Efraín Velastegui López. ${ }^{4}$

\section{DOI: https://doi.org/10.33262/anatomiadigital.v4i2.1719}

\begin{abstract}
.
Resumen.

The equine therapy is a treatment form La equinoterapia es una forma de for people with diverse pathological tratamiento para personas con diversos processes that it uses as therapeutic element the patient's relationship procesos patológicos, que emplea como elemento terapéutico la relación del affected with the horse, so much in it paciente afectado con el caballo, tanto en mounts it as in the care of the animal, la monta como en el cuidado del animal, and that it takes advantage of the movement multidimensional of the horse y que aprovecha el movimiento multidimensional del caballo con un

\footnotetext{
1 Facultad de Cultura Física de Mayabeque Departamento Educación Física, Mayabeque, Cuba. josedv@unah.edu

2 Facultad de Ciencias Médicas de Mayabeque, Departamento Cultura Física, Mayabeque,

Cuba, ergonzales@infomed.sld.cu

${ }^{3}$ Centro de Rehabilitación "Luis Li Trijent", Especialistas Medicina Física y Rehabilitación y Medicina General Iintegral, luisamaria@infomed.sld.cu

${ }^{4}$ Ciencia Digital Editorial, Ecuador, luisefrainvelastegui@cienciadigital.org
} 
with a therapeutic sense. The therapeutic sense of the activity comes given by the form in that the professional uses the horse, for the individuality of the actions that develops in relation to each patient's specific characteristics and the evolutionary moment in that she is. The main objective is that they can have an experience gratificante and positive that helps them to improve its quality of life and its personal well-being. It is unfavorable on the other hand that a great part of the professionals of the health and the special education ignore the foundations of this therapeutic modality that it could be a complementary alternative element of value to the activities that daily the children disability develop. At the present time many institutions in charge of promoting the infantile rehabilitation don't include the equine therapy inside their recommendations. This activity of social interest has been little studied from the angle of its therapeutic value what outlines a Problem to the Science and the Technology for its importance for Cuba, an underdeveloped country that he/she has been able to introduce novel technologies in the environment of the health in spite of the provoked lack of resources for more than 61 years of blockade.

Keywords: $\quad$ Equine therapy, Palabras claves: Equinoterapia, Rehabilitation, Science, Technology, Rehabilitación. Ciencia, Tecnología, Horses, Disability Children sentido terapéutico. El sentido terapéutico de la actividad viene dado por la forma en que el profesional emplea al caballo, por la individualidad de las acciones que desarrolla en relación a las características específicas de cada paciente y el momento evolutivo en que se encuentra. El objetivo principal es que puedan tener una experiencia gratificante y positiva que les ayude a mejorar su calidad de vida y su bienestar personal. Resulta desfavorable por otra parte que una gran parte de los profesionales de la salud y la educación especial desconocen los fundamentos de esta modalidad terapéutica, que pudiera resultar un elemento alternativo complementario de valor a las actividades que diariamente desarrollan los niños discapacitados. En la actualidad muchas instituciones encargadas de promover la rehabilitación infantil no incluyen la equinoterapia dentro de sus recomendaciones. Esta actividad de interés social ha sido poco estudiada desde el ángulo de su valor terapéutico lo que plantea un Problema a la Ciencia y la Tecnología por su importancia para Cuba, un país subdesarrollado que ha podido introducir novedosas tecnologías en el ámbito de la salud pese a la carencia de recursos provocada por más de 61 años de bloqueo. Sociedad, Caballos, Discapacidad, Niño. 


\section{Introducción}

La salud de la población es un problema objeto de estudios por las Ciencias Sociales en el contexto de relación entre lo biológico y lo social, a partir del proceso contradictorio que revela la salud-enfermedad de los individuos como seres bio-psico-sociales, que se expresa en la complejidad de las contradicciones del conjunto de las relaciones sociales.

Toda esta situación terrible que afecta en gran medida la salud de la humanidad por el mal uso de la ciencia y la tecnología en los países del primer mundo, lleva a que muchos renieguen del pensamiento crítico y asuman una posición nihilista del lenguaje simbólico de la cultura neoliberal. Otros adoptan una posición academicista y se desgastan en foros y tribunas de una supuesta reivindicación de la teoría por la teoría, sin tener como fin la solución de los grandes problemas sociales que hoy enfrenta la humanidad, y en particular los de la salud.

En medio de esa situación desde hace más de 60 años se da a conocer al mundo un procedimiento alternativo novedoso en el campo de la terapia a niños: La Equinoterapia.

En el incesante esfuerzo por mejorar la calidad de vida de las personas discapacitadas la ciencia a través de la historia de la medicina, ha empleado con mayor o menor éxito innumerables procedimientos terapéuticos, generalmente utilizando medica medicamentos y fisioterapia, por tanto la equinoterapia se incorpora al conjunto de esos procedimientos como forma novedosa para influir en desarrollo efectivo de las terapias recuperativas de los pacientes discapacitados y de enfermedades asociadas al sistema nervioso central, entre otras.

El desarrollo actual de la ciencia coloca al médico en una situación especial, la práctica de la profesión debe hoy más que nunca estar basada en un riguroso espíritu de objetividad científica.

Por tanto la recomendación de empleo de un determinado procedimiento debe partir de la "evidencia científica' que la misma será realmente beneficiosa al paciente, elemento que requiere integrar la experiencia clínica con los resultados de las investigaciones.

Desde otro ángulo del análisis, el desarrollo de la informática en los últimos años ha puesto a disposición de los profesionales de la salud, una amplia gama de reportes sobe estudios relacionados con el empleo de la equinoterapia en la atención al niño discapacitado, aunque algunos de ellos por lo pequeño de la muestra no permiten establecer conclusiones definitivas, aunque de forma general se considera beneficioso este proceder terapéutico. Win Barolin pionero de la equinoterapia en Austria publicaba en 1991, que los favorables resultados alcanzados durante varios años de empleo de este proceder, destacan el impacto positivo que tiene en el ajuste psicológico de los pacientes y reportaba cambios favorables en la conducta de un grupo de niños con dificultades sensoriales después de la equinoterapia. 
Los actuales estudios sobre este tema revelan los indiscutibles beneficios que se alcanzan con la práctica de la equinoterapia, proceder que pudiera generalizarse mediante el empleo complejo con otras técnicas terapéuticas convencionales que no emplean el caballo, pero es evidente que con las técnicas convencionales el niño no experimenta sentimientos de diversión, el placer y la alegría que le proporciona la actividad controlada de la monta del caballo, además de la estrecha relación afectiva que establece con el animal.

En algunos casos niños discapacitados son privados de participar en actividades al aire libre y de jugar con otros niños, la práctica de la equinoterapia les crea un significado especial en cuanto a la comunicación social fuera del ámbito familiar, que constituye otra ventaja ante otros procederes.

La equinoterapia al igual que sucede con la acupuntura, la quiropraxia y otros muchos procederes alternativos, va ganando progresivamente espacio entre los profesionales dedicados a la atención de los niños discapacitados. Hace 20 años si alguien sugería montar un niño discapacitado en un caballo, inmediatamente la respuesta era que esta actividad además de ser peligrosa no determinaba ningún beneficio, hoy en cambio, son muchos los centros de salud del país que recomiendan su uso de forma sistemática por su impacto en el desarrollo psico-social y comunicacional de los discapacitados con resultados beneficiosos.

Los argumentos anteriores permiten expresar como objetivos del presente trabajo los siguientes:

Explicar la importancia de los estudios de equinoterapia como alternativa dirigida al tratamiento de la discapacidad infantil, como un proceso de restablecimiento terapéutico real, necesario y objetivo que debe tener un impacto social positivo para la recuperación de la salud de niños y otras patologías en general existentes en nuestro país.

Valorar el impacto que debe tener para la sociedad el uso de la equinoterapia como proceder terapéutico sustentado en la ciencia aplicada al tratamiento de la discapacidad Infantil.-Generalizar experiencias sobre estudios vinculados a la equinoterapia que contribuya a crear conciencia y cultura en la sociedad, sobre la necesidad de incorporarla como terapia efectiva para el restablecimiento de niños discapacitados.

\section{Metodología}

En la medicina tradicional China, se hace referencia al valor de la equitación para el tratamiento de diversas condiciones de salud. Hipócrates de Cos, la recomendaba para el tratamiento de dolencias como el insomnio y enfermedades musculares. Asclepíades de Prusia consideraba que el movimiento del caballo mejoraba el estado de salud de pacientes afectados por la gota o la epilepsia. Galeno, recomendaba la equitación para mejorar el estado de salud y prevenir la aparición de enfermedades. Merkurialis (1569) planteaba que montar a caballo ayudaba a conservar una buena forma física. Thomas 
Sydenham (1689) en su libro sobre el tratamiento de la gota, proponía la práctica de la equitación como una actividad que mejoraba los síntomas y evitaba las crisis. ${ }^{1}$

Otros autores como: Francisco Fuller (1704), Frieddrich Hoffman (1719), George E. Stahl (1734), John Pringle (1752), Samuel T. Quelmalz (1758), Giuseppe Benvenuti (1772), Joseph C. Tissot (1782), quienes ejercieron la práctica de la medicina, consideraban la equitación como una actividad a favor de la salud tanto física como psíquica. $^{2}$

Todos los estudios realizados revelan que en el ámbito de la práctica médica vinculada a procederes de restablecimiento de la salud, los investigadores observaron diversos beneficios del empleo de la equitación, aunque los argumentos utilizados estaban en correspondencia al desarrollo de la ciencia de la época y algunas conclusiones, se correspondían con el bajo nivel de conocimientos existente de las causas y efectos que tenían diversas enfermedades en el desarrollo psicomotor y psico-social de los infantes.

La primer referencia del empleo de la equitación vinculada con el proceso de convalecencia de pacientes ingresados en un hospital, se remonta a Inglaterra en 1901, en el Hospital Ortopédico de Oswentry y más tarde en 1917 en el Hospital Universitario de Oxford, donde se atendían heridos de la primera Guerra Mundial y se utilizaba la equitación como parte del proceso de rehabilitación de los lesionados.

El empleo de la equinoterapia se consolida a partir de la Segunda Guerra Mundial, en particular en Alemania. Un pionero en el empleo de la técnica lo fue el doctor Max Reichenbach (1953), quien describió por primera vez, los fundamentos teóricos de su uso en los programas de rehabilitación. Progresivamente la equinoterapia se extiende a Francia, Inglaterra, Canadá y otros muchos países.-En 1956 fue creada la primera asociación en Inglaterra. En Francia la reeducación ecuestre desde el ángulo médico nació en 1965.

El primer Congreso Internacional sobre equinoterapia se realiza en 1974, en Francia. En 1985 en la ciudad de Milán, Italia, se creó la Federación Internacional de Equinoterapia (Federation Riding for the Disabled International -FRDI). ${ }^{3}$

Un ejemplo que influyó favorablemente en la generalización de la práctica de la equinoterapia lo constituye el caso de Liz Hartel (Dinamarca), una adolescente que a la edad de 16 años fue afectada por la poliomielitis y quedó con severas limitaciones para caminar, pero a pesar de sus limitaciones compitió en la Olimpiada de 1952 y ganó la medalla de plata en la competencia de adiestramiento, y repitió la hazaña en $1956 .{ }^{4}$

A partir de la década de 1960, la equinoterapia adquiere una nueva dimensión cuando los profesionales vinculados con su empleo, se esfuerzan por reunir evidencia científica que permitiera brindar argumentos válidos a la comunidad científica médica sobre el valor de la técnica y en 1969, en el Centro Hospitalario de Salpetriére en París, se presenta el primer trabajo científico sobre reeducación ecuestre y en 1971 se defiende la primer tesis de doctorado sobre el tema. ${ }^{5}$. 
En América Latina, a partir de la década de 1980, toma auge la actividad en países como Brasil, México y Argentina y en 2003, se suma Venezuela. En la actualidad la técnica se aplica con mayor o menor profesionalidad en todo el continente americano, y en la mayoría de los países existen organizaciones que promueven el empleo de la equinoterapia con mayor o menor éxito.

La década del 2000 al 2010 determinó un enfoque más científico basado en el empleo de modernas tecnologías en el estudio de los beneficios del empleo de la equinoterapia. El desarrollo de la neurociencia y la mejor compresión de la influencia del medio sobre el desarrollo del sistema nervioso, aportaron argumentos sobre el impacto del movimiento rítmico del caballo en la rehabilitación de las secuelas neurológicas.

Hammer y $\operatorname{otros}^{6}$ reportan los beneficios de la práctica de la equinoterapia en el ajuste emocional y equilibrio en pacientes afectados por esclerosis múltiple, lo favorable del clima permite que la actividad se desarrolle todo el año al aire libre, en un contexto peculiar lleno de colores, formas, sonidos, olores, plantas, que resulta altamente atractivo para el que asiste a recibir tratamiento y representan un elemento facilitador en la relación entre el jinete y el caballo. Si se compara este tratamiento en un ambiente favorable con las técnicas convencionales que se emplean en la sala de un hospital o de un centro de rehabilitación, las diferencias son evidentes.

Los estudios en Cuba se inclinan en lo particular a considerar los beneficios de combinar la equinoterapia a partir de la evaluación individual de la forma de expresión del síndrome invalidante, con otras técnicas de rehabilitación desarrolladas en el contexto natural del centro de equitación, entre ellas: fisioterapia, terapia ocupacional, educación especial, logopedia, arte-terapia, intervención familiar y comunitaria. Prácticas de amplio dominio en el sector deportivo cubano.

Un argumento a favor de la equinoterapia desde nuestro punto de vista, es que entre jinete y el caballo se produce una comunicación extra verbal, sucede un intercambio de información sensorial y auditiva nueva, el caballo percibe al jinete y sus características, y estas influyen en su estado emocional y desempeño, de igual forma el jinete reconoce las características del animal y le genera respuestas complejas organizadas en su sistema nervioso. No hay un equipo de mecanoterapia en un departamento de fisioterapia que pueda reproducir la compleja estimulación del movimiento que produce el caballo con su desplazamiento tridimensional.Variados son los ejercicios que pueden realizarse sin la participación del caballo, pero la mayoría son mucho menos divertidos para el niño que los que se realizan en el curso de la equinoterapia.

La equinoterapia en Cuba se inició como una buena idea, defendida con energía y entusiasmo por un grupo de personas con la visión de lo que podía ayudar la actividad curativa e incrementar la calidad de vida y el desempeño funcional de los discapacitados.

En relación a la equinoterapia, es imprescindible un desarrollo organizado, científicamente documentado, planificado, que parta en primer lugar de la capacitación 
de los profesionales que van a emplear el procedimiento y la garantía de los recursos imprescindibles para un uso eficaz y seguro.

Precisamente los problemas a enfrentar desde el ángulo de los recursos a destinar y el costo del proceder resulta un problema social en Cuba, donde las limitaciones del bloqueo norteamericano por un lado y la falta de estudios sistematizados sobre el tema, limitan el desarrollo de los conocimientos científicos sobre el tema, lo que afecta la necesaria contribución de los esfuerzos a nivel territorial o sectorial, para armonizar las complejas interrelaciones entre la ciencia, la tecnología y la solución de este problema social.

En el sentido amplio del análisis se puede apreciar que el problema social de la ciencia y la tecnología en el caso del tema de la equinoterapia, no constituye un fenómeno nuevo analizado por la Ciencias Sociales, sino que tiene su génesis en las mismas condiciones socio-históricas, económicas y políticas que tiene que enfrentar Cuba como país subdesarrollado,

Es una práctica universal que se reservan para sí y para los que tienen recursos financieros suficientes -sin incluir el bloqueo que lo agrava- para acceder a la producción científica y de bienes intensivos de tecnología, lo que deriva entonces en uno de los grandes problemas sociales que hoy día enfrenta la humanidad para su desarrollo.

En realidad, la importación de tecnologías por parte de los países subdesarrollados, lejos de permitir la obtención de los objetivos del desarrollo esperado, se ha venido a sumar al conjunto de los factores que refuerzan la situación de dominación extranjera que sufren la mayoría de los países del Tercer Mundo y contribuye a la reproducción del atraso y el subdesarrollo. Esto constituye otro de los grandes problemas sociales que enfrenta la humanidad.

Por otra parte, los países subdesarrollados tienen que enfrentar la elevación considerable en los costos de las tecnologías importadas debido a una mayor pretensión de los derechos de propiedad intelectual a nivel internacional. Los gastos en inversión y desarrollo y su adecuada asignación entre los distintos sectores socio-económico son factores determinantes para competir con la posibilidad de éxito en un mercado mundial altamente sensible a los últimos adelantos de la ciencia y la tecnología. Por lo que esto constituye otro de los grandes problemas sociales que enfrenta la humanidad provocado por su mal empleo en determinados países.

Otro de los grandes PSCT se enmarca en que los países industrializados invierten en investigación y desarrollo unos ciento noventa y cinco mil millones de dólares anuales, en tanto los países subdesarrollados, apenas unos 10 mil millones de dólares por año, y es donde reside las tres cuarta parte de la población mundial.

El desarrollo de la Ciencia y la tecnología necesita de un replanteo del modo tradicional de enfocar la solución de sus problemas, enfocado en las necesidades de los pueblos del 
sur, pues se han producido grandes descubrimientos en el campo de lo biológico y hasta químico, de fenómenos y procesos que involucran la salud del hombre en su integración con la naturaleza y su acción social, que en los países capitalista se ocupan de degradar hasta límites de hacer desaparecer la especie humana, como expresara Fidel en la conferencia mundial sobre medio ambiente en 1992. De ahí surge un pensamiento biótico, de conciencia ecológica y una nueva cultura que se abre paso con grandes desafíos para la sobrevivencia de la humanidad.Existen varios factores que han atentado y atentan contra el desarrollo de la ciencia y se dan por igual en todos los países subdesarrollados en mayor o en menor medida.

Entre ellas tenemos la enajenación de la ciencia con la producción, una consecuencia de esto ha sido la baja capacidad de producción científica y de generación de tecnología. La polarización del sistema científico técnico internacional, este está dividido en dos polos, en el primero recae el peso y la orientación de la ciencia, en el otro la debilidad de las instituciones científicas de los países subdesarrollados, esto no es una situación conyugal sino estructural que se consolida y ahonda, lo que consolida la tesis de que la polarización es una propiedad estable del sistema científico internacional. El ambiente desfavorable en lo político y económico desestimulo la ciencia e incentiva la fuga de cerebros.

La investigación original se subvalora, la comunidad local no confía lo suficiente en sí misma y busca los criterios de valoración en el exterior, se pierde el interés de sus miembros en comunicarse entre ellos, sobre todo los de más alta calificación que tienen acceso fácil al medio internacional, el investigador suele sufrir el reproche social por la escasa contribución que hace a la solución de los problemas del subdesarrollo, sin que esté garantizada la demanda social de su posible contribución.

También se habla del robo de cerebro en el sentido de una orientación exógena del trabajo científico endógeno, en buena medida el sistema de producción de conocimiento en los países latinoamericanos están determinados por patrones científicos, criterios y selección de problemas que provienen del exterior.

La Revolución Científico-Técnica, tanto por su influencia sobre el desarrollo social en general como sobre el desarrollo de las Ciencias Médicas, ha representado una posibilidad extraordinaria de progreso para el proceso salud en el hombre. Esto de concreta en la mayoría de los indicadores como el aumento de la expectativa de vida, la sensible disminución de la morbilidad de enfermedades infecciosas y las enormes posibilidades que tienen a manos estas ciencias que no las tenían en el siglo pasado, ha mejorado las comunicaciones con las nuevas tecnologías de punta, el conocimiento cada día mayor del hombre en cuanto a los secretos de la realidad objetiva, el conocimiento de patologías desconocidas hasta entonces que afectaban la salud del ser humano y que hoy han tenido solución por la Ciencias Médicas.

Un componente de obligada referencia dentro del indicador de calidad de vida y el nivel de vida de la salud humana, por lo que los avances tecnológicos más recientes en el 
ámbito particular de los servicios médicos constituye un elemento de vital importancia. Sin embargo, el desarrollo desproporcionado y la brecha existente entre los países desarrollados y subdesarrollados, hacen que este importante componente de calidad de vida sea una falacia para una gran parte de la población de mundo.

El alto costo de estas tecnologías encarece la atención médica sosteniblemente en los últimos años y establece el dilema de quiénes deben beneficiarse principalmente de ellos, los que pueden pagar, o los que tienen la sintomatología adecuada para su uso más eficaz. Estas técnicas se emplean indiscriminadamente y ocasionan, en no pocos casos, cierta indisposición del paciente que se ve sometido a un procedimiento caro y resultados poco convincente o conocido.-Por otra parte, el problema de la información científica cada vez se muestra más candente en la medida en que las potencias dominan la producción y la diseminación de ésta con una franca tendencia a circular en una misma dirección.

Los países subdesarrollados consumen fundamentalmente una información elaborada por los países desarrollados, lo que entraña una forma de dominación sutil pero terriblemente eficaz, ya que tienen el acceso a lo más novedoso en las Ciencias Médicas para el tratamiento a los pacientes.

A menudo la alta tecnología se importa de otra nación más desarrollada y se utiliza sin tener en cuenta las características locales y en ocasiones no están acompañadas por un uso justificado y por el desarrollo de un personal calificado y como consecuencia se presenta dificultades en el mantenimiento, los resultados no son enteramente satisfactorios.-Y no obstante cada año los precios aumentan en proporción muy superior al costo de la vida, convirtiéndose esto en un grave problema social.

Gran parte de los problemas que aquejan al mundo actual, en materia de salud por el mal uso de la ciencia y la tecnología, podrían resolverse fácilmente si estas se pusieran al alcance de todas las sociedades de forma noble y justa.

Cuba es un país del Tercer Mundo, pero comparte muchos de las soluciones a los problemas sociales que hoy aquejan a la humanidad, derivados del mal uso de la ciencia y la tecnología por los países ricos, sobre todo en el plano de la salud por las políticas imperiales. Si se pretende evaluar el estado del desarrollo de nuestros países y las alternativas de su desenvolvimiento, resulta de obligado análisis la situación científico-tecnológica no sólo por su importancia para el proceso de desarrollo en la industrialización, sino también para la transformación cualitativa de todas las esferas de la vida social ya que todas tienen un carácter social.

Gracias a las conquistas sociales de la Revolución Socialista, se puede hacer más con menos de forma eficiente. Se cuenta con el potencial humano necesario para ello, y tenemos el principal, el potencial científico. Se dispone de científicos y técnicos al servicio de la salud del pueblo, de la satisfacción de las grandes necesidades, que se conjugan con las proféticas palabras del Líder indiscutible de la Revolución cuando 
dijera en la década del 60 que "El futuro de este País ha de ser un futuro de hombre de ciencias..."

En este contexto, el diseño y aplicación de la política de salud cubana refleja un significativo esfuerzo encaminado a enfrentar las dificultades en virtud de la voluntad política que priorice el nivel de salud de la población. La política de crear centros donde se materialicen la investigación, el desarrollo y la producción, es el resultado de una filosofía nueva y prometedora, que reconoce la necesidad de desarrollar una capacidad tecnológica endógena no solo para asimilar y adoptar la nueva tecnología impartida en condiciones apropiadas, sino también hacer frente a las necesidades del país.

Por tanto, no existe ninguna tecnología aún la más sofisticada a las que no acceda la organización de la salud pública cubana, y por tanto, toda la población, lo que se pone de manifiesto entre los logros más importantes del desarrollo científico-tecnológico. De esta forma Cuba ha alcanzado niveles de salud comparable a los países más desarrollados.

\section{Resultados}

Un desafío a las ciencias médicas lo constituyen las sustancias nocivas y desechos en general vertidos al medio ambiente, producto de la actividad productiva humana, que constituye una agresión constante al medio ambiente con el resultado de que la biosfera ha ido perdiendo su capacidad de autodepuración y reciclaje natural.

El hombre, sin proponérselo, por el mal uso de la ciencia y la tecnología en correspondencia al sistema social, ha alterado los ciclos naturales del planeta que habita, ha roto o interrumpido su equilibrio ecológico, con todas las consecuencias negativas para el conjunto de los seres vivos, incluía la salud del hombre.

Relacionando la presente investigación con las transferencias de tecnología, la innovación y la racionalización que se han realizado para la sustitución de tecnología se puede referir que una de ellas está encaminada al tratamiento de diversas patologías mediante la hipoterapia activa y pasiva.

En este proceder está presente la innovación y la racionalización de los recursos que se ponen al servicio de esta terapia en beneficio de los niños discapacitados. Entre los resultados logrados están la silla de montar, para el tratamiento de equinoterapia en los pacientes discapacitados, por la necesidad de resolver la montura con características adecuadas a las de este tipo de paciente y ajustándonos a las exigencias del personal calificado en esta tarea y teniendo en cuenta que para la rehabilitación de estos niños, se debe estar lo más pegado posible a la piel del caballo. Se debe destacar que dicha montura se realiza no con los materiales propios de una silla normal sino con los que han sido capaces de hacer en nuestro país, debido a su alto costo por unidad en el extranjero. 
Valorando la necesidad de este aditamento se han realizado otras mejoras que permiten desarrollar algunas características importantes en este tipo de terapia y analizar sus resultados en el uso de la montura que se les brinda a los pacientes, ofreciéndoles una solución inmediata para así resolver esta dificultad existente en nuestro país.

Además se han creado medios para sustituir equipos como algunos de los instrumentos que se emplean con más frecuencia, entre ellos las bañeras para el masaje subacuático, bañeras de hidromasajes, bañeras electro acuáticas, y bañeras médicas entre otras, esto es para tratamientos de hidromasajes, además de mesas de verticalizaciòn, aparatos de gimnasia con resistencia flexible y dispositivos para ejercicios en la suspensión y descarga, materiales y equipos para terapias ocupacionales cognoscitivas orientadas a la atención como memo fotos de objetos, alimentos y animales de mundo, así como asociación con animales, colores, imágenes secuenciales entre otros, para el tratamiento con niños autistas y de déficit de la atención, juegos de destreza para a psicomotricidad y otros.-Es curioso observar que muchos de los equipos creados en Cuba, a pesar de su bajo costo, sean diseñadas en países con alto desarrollo científico-técnico (Estados Unidos, Canadá, Alemania, Inglaterra, Francia, etc.) con precios más de 10 veces mayores a los cubanos.

Esto es debido a que precisamente en estos países es donde se encuentran los instrumentos necesarios, la información más reciente y el personal calificado para llevar a cabo estas investigaciones y producir estos equipamientos que ofertan no para la salud del ser humano, sino para la salud del mercado que representan.

Se debe expresar que la equinoterapia pese a la carencia de recursos motivadas por el bloqueo y pese a los grandes esfuerzos que hacen todos los centros habilitados del país, para poder continuar el trabajo de la rehabilitación mediante esta terapia con equinos y los resultados positivos en la mejoría de la calidad de vida de los niños, aún es insuficiente su desarrollo como terapia adecuada a los incapacitados y requiere de mayor cultura e integración social, para lograr resultados superiores del aporte científico, que beneficiará entre otros: a los perfiles de la profiláctica y terapéutica en el país, ayudando en la formación de nuevos profesionales, promover la igualdad de género, reducir la tasa de mortalidad infantil.

Los aspectos negativos en la carencia de recursos como equipamiento para equinos, sillas, mantas, paños y cascos para montar, entre otros, y los equipos para el trabajo de motricidad y psicomotricidad para las diferentes patologías, intercambio de información con centros especializados en el mundo, la creación de clínicas para equinos, constituyen la vía para no tener que gastar cuantiosos recursos en adquirir equipos y medicamentos costosos en mercados lejanos debido al Bloqueo.

En la equinoterapia terapia está presente la multilateralidad de un conjunto de ciencias como las médicas, Veterinarias, Pedagógicas, Psicológicas, Logopedia, Biomecánica médica, traumatología, ortopedia, gimnasia terapéutica, Cultura física, fisiología, Morfología, Rehabilitación física, entre otras que conforman un equipo 
multidisciplinario y que tienen que estar altamente preparados desde el punto de vista profesional.

Todo hombre de ciencia ha de regirse por la regla de oro de quien produce conocimiento o sea: "Todo lo que hago debe tener una finalidad humanista". Así como ser responsable de la investigación desde el inicio hasta su aplicación y mantener en todo momento su ética, que incluye honestidad, veracidad, responsabilidad, ponderación, justicia, modestia y patriotismo.

\section{Conclusiones:}

- La equinoterapia constituye una alternativa viable y eficaz para el manejo rehabilitador en niños con discapacidad infantil. Esta experiencia no sólo es enriquecedora para los niños sino también para los padres y demás personas relacionadas con ella, entre otros aspectos que se destacan en este tipo de actividad, dada en la mejor comprensión hacia las personas con discapacidad y el reconocimiento de su valor como seres humanos por parte de los demás, lo que contribuye a una mejor integración social de las mismas.

- En las condiciones del mundo globalizado en que vivimos hoy, el uso de estos conocimientos en el proceso salud-enfermedad resulta un gran impacto para la sociedad, puesto que crea grandes asimetrías en cuanto al acceso a los medios que se utilizan para el tratamiento.

- Cuba realiza grandes esfuerzos para contribuir con la salud de estos pacientes, pero como país del Tercer Mundo también adolece de recursos materiales imprescindibles para llevar a todos los municipios estos procederes terapéuticos, determinantes en la salud de los niños con estos tipos de patologías.

- La solución a ese problema social es la realización investigaciones orientadas a buscar tecnologías endógenas y equipamientos que sustituyan importaciones, así como trabajar de conjunto con los territorios para aportar determinada de masa de equinos destinada a la terapia y las investigaciones, con el fin de lograr mayores resultados científicos y generalizar su empleo a nivel nacional.

\section{Referencias bibliográficas}

Edith Gross Naschert. Equinoterapia la rehabilitación por medio del caballo _ México _ 2006, Editorial Trillas.

Potter JT, Evans JW, Nolt BH Jr. Therapeutic horseback riding. J Am Vet Med Assoc1994 Jan 1;204(1):131-3 
Pérez Álvarez L. La equinoterapia en el tratamiento de la discapacidad infantil. Revista Archivo Médico de Camagüey. Vol. 12. No. 1. Año 2008.

Edith Gross Naschert. Equinoterapia la rehabilitación por medio del caballo _ México _ 2006, Editorial Trillas.

Organización Mundial de la Salud. Clasificación Internacional del Funcionamiento de la Discapacidad y la Salud, 2004.

Memorias del XII Congreso Internacional de Equitación Terapéutica desarrollado en Brasil en el 2006

Davis E, Davies B, Wolfe R, Raadsveld R, Heine B, Thomason P, Dobson F, Graham HK.A randomized controlled trial of the impact of therapeutic horse riding on the quality of life, health, vand function of children with cerebral palsy. Dev Med Child Neurol. 2009 Feb;51(2):111-9.

Ercilla Arana M. y Espinoza Valdés R. (2006) Tecnología apropiada: concepción para una cultura. Editado en Tecnología y sociedad por GEST Pág. 19

Gross Naschert. E. (2006) Equinoterapia la rehabilitación por medio del caballo _ México _, Editorial Trillas.

Lecturas de filosofía Salud y Sociedad. Colectivo de Autores. Editorial de Ciencias Médicas La Habana 2000

Medicina General Integral. Volumen 1. Editorial de Ciencias Médicas. La Habana 2001

Núñez Jover, J. (1999) La ciencia y la tecnología como procesos sociales., Editorial Félix Varela, La Habana.

Potter JT, Evans JW, Nolt BH Jr. Therapeutic horseback riding. J Am Vet Med Assoc1994 Jan 1;204(1):131-3.

Reynel A Llames Belett. Glosario Filosófico. Editorial de Ciencias Médicas. La Habana 2007.

Reynel A Llanes Belett. Lecturas Complementarias para profundizar y actualizar los conocimientos de Filosofía. Editorial de Ciencias Médicas. La Habana. 2011. 


\section{PARA CITAR EL ARTÍCULO INDEXADO.}

Díaz Valdés, J., González Pérez, E. R., Torres García, L. M., \& Velastegui López, L. E. (2021). La equinoterapia como una alternativa terapéutica dirigida al tratamiento de la discapacidad infantil y su impacto en el proceso de rehabilitación. Anatomía Digital, 4(2), 181-194. https://doi.org/10.33262/anatomiadigital.v4i2.1719

\section{Ciencia \\ LDigital}

El artículo que se publica es de exclusiva responsabilidad de los autores y no necesariamente reflejan el pensamiento de la Revista Anatomía Digital.

El artículo queda en propiedad de la revista y, por tanto, su publicación parcial y/o total en otro medio tiene que ser autorizado por el director de la Revista Anatomía Digital.
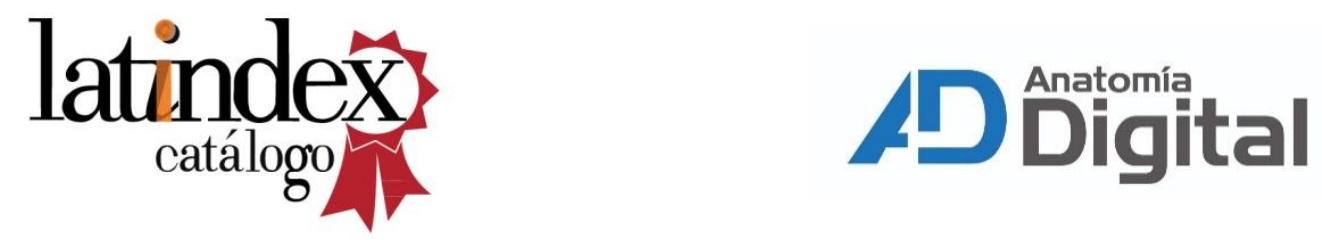